\title{
Surgical versus conservative therapy for multiple rib fractures: a retrospective analysis
}

\author{
Yongjing Liu, Shun Xu, Qi Yu, Yu Tao, Leilei Peng, Shengbo Qi, Hao Han, Mengran Chen \\ Department of Cardiothoracic Surgery, The 105th Hospital of Chinese People's Liberation Army, Hefei 230031, China \\ Contributions: (I) Conception and design: Y Liu; (II) Administrative support: Y Liu; (III) Provision of study materials or patients: Y Liu, S Xu; (IV) \\ Collection and assembly of data: Y Liu, S Xu; (V) Data analysis and interpretation: Y Liu, S Xu; (VI) Manuscript writing: All authors; (VII) Final \\ approval of manuscript: All authors. \\ Correspondence to: Yongjing Liu. Department of Cardiothoracic Surgery, The 105th Hospital of Chinese People's Liberation Army, Hefei 230031, \\ China. Email: yongjingliu@163.com.
}

Background: In this study, we aimed to evaluate the clinical effects of multiple rib fracture treatments using a rib plate fixator.

Methods: From June 2014 to December 2016, 110 cases of patients with multiple rib fractures were collected for our study from the 105th Hospital of PLA; 59 patients were treated by surgery, and 51 patients received conservative treatment. We compared surgical rib plate fixation with conservative treatment for patients with multiple rib fractures.

Results: The hospital stay for the surgical group was $13.12 \pm 4.21$ days, whereas for the conservative group, it was $18.57 \pm 5.39$ days $(\mathrm{P}<0.001)$. The duration of the thoracic intensive care unit (ICU) stay was $4.02 \pm 1.41$ days in the surgical group and $5.06 \pm 1.80$ days in the conservative group $(\mathrm{P}=0.001)$. The indwelling thoracic drainage tube time was $5.85 \pm 1.52$ days in the surgical group and $8.26 \pm 1.96$ days in the conservative group $(\mathrm{P}<0.001)$. The index of partial pressure of arterial oxygen $\left(\mathrm{PaO}_{2}\right)$ was $91.05 \pm 10.88 \mathrm{mmHg} 24 \mathrm{~h}$ after surgery whereas $86.49 \pm 11.69 \mathrm{mmHg} 24 \mathrm{~h}$ after conservative treatment $(\mathrm{P}=0.036)$. The index of partial pressure of carbon dioxide $\left(\mathrm{PaCO}_{2}\right)$ was $37.80 \pm 2.86 \mathrm{mmHg} 24 \mathrm{~h}$ after surgery and it was $39.08 \pm 2.46 \mathrm{mmHg}$ $24 \mathrm{~h}$ after conservative treatment. The hospitalization cost was $6,206.44 \pm 371.42$ USD for the surgical group and 4,544.61 \pm 524.79 USD for the conservative group $(\mathrm{P}<0.001)$. The number of rib displacement cases after treatment was 1 case in the surgical group and 2 cases in the conservative group $(\mathrm{P}=0.475)$. Atelectasis occurred in 10 cases in the surgical group and in 17 cases in the conservative group $(\mathrm{P}=0.046)$. Delayed hemopneumothorax occurred in 7 cases in the surgical group and in 16 cases in the conservative group $(\mathrm{P}=0.012)$.

Conclusions: Surgical treatment is a safe and effective therapy for multiple rib fractures. Our data suggested that surgical treatment can significantly shorten the patients' pain-endurance time, hospitalization time, and can reduce complication incidence. Severe rib fracture patients might benefit from surgical fixation.

Keywords: Plate fixator; multiple rib fractures; surgical

Submitted May 27, 2018. Accepted for publication Sep 25, 2018.

doi: $10.21037 /$ atm.2018.10.12

View this article at: http://dx.doi.org/10.21037/atm.2018.10.12

\section{Introduction}

Thoracic trauma is a major traumatic injury throughout the world, and it has very high incidence in developing countries (1). Thoracic trauma is often associated with significant morbidity and mortality $(2,3)$. Most patients with thoracic trauma do not undergo surgical treatment. However, some cases are complicated by serial rib fractures (4). Without effective treatment, multiple fractures 
of multiple ribs can cause the partial chest wall lose the ribs to support, and also causes paradoxical breathing, the softening area of chest wall invagination when inhaling, and outer protrusion when exhaling, it is called the flail chest (5), can cause long-term disability, chest wall pain, and the paradoxical motion of the chest wall inducing respiratory distress (6).

The recent goals of multiple rib fracture treatment are to eliminate the symptoms of pain and breathing difficulty, and to recover thoracic stability. Different treatments for rib stabilization have been reported in recent years (7); Therefore, the goals of surgical treatment are to stabilize the chest wall, however, the treatment of rib fractures remains controversial $(8,9)$. Conservative treatment is generally associated with safety and lower hospitalization costs, although surgical treatment can correct the thoracic deformity better $(10,11)$. In addition, with the development of biomedical engineering, various types of rib plates can be chosen for internal fixation surgery of multiple rib fractures.

In this research, we review the main clinical indexes of different treatment to multiple rib fractures, and compare the particular features of the two treatments. In addition, we introduce the visual analog scales (VAS) scores to compare the level of pain between surgical group and conservative group.

\section{Methods}

\section{Rib fractures of patients}

We collected the clinical data of 390 patients with serial rib fractures who received medical treatment at the 105th hospital of the Chinese People's Liberation Army. In total, 70 patients received surgical treatment, and 320 patients received conservative treatment. We performed a retrospective study: 59 cases were grouped into a surgical treatment group, and 51 cases were grouped into a conservative group. In addition, the rib fractures were caused by a variety of factors, including road traffic trauma, fall injury, blunt instrument injury and crush injury. All the patients received thoracic computer tomography scanning. Patients received surgical treatment within $24 \mathrm{~h}$ of admission in the surgical group. We compared the difference between the two groups by the following measures: hospital days, thoracic intensive care unit (ICU) stay days, indwelling thoracic drainage tube time, fracture displacement after admission, atelectasis after medical treatment, delayed hemopneumothorax, pain scores according to VAS, hospital costs, partial pressure of arterial oxygen $\left(\mathrm{PaO}_{2}\right)$ of arterial blood $(24 \mathrm{~h}$ after surgical treatment or conservative treatment), and partial pressure of carbon dioxide $\left(\mathrm{PaCO}_{2}\right)$ of arterial blood $(24 \mathrm{~h}$ after surgical treatment or conservative treatment). This is a retrospective study, and the study was authorized by the hospital's ethics committee (2017L16). All the 110 patients had clear clinical indications for therapy.

\section{Emergency treatment of the patients}

For the 110 cases associated with serial rib fractures, electrocardiogram monitoring and hyperoxia intake were pursued to monitor the vital signs and provide easier breathing for the patients, respectively. There were 18 open chest trauma patients, and the chest trauma was sutured in the emergency treatment. Closed thoracic drainage was performed for the patients with severe hemothorax and/or pneumothorax. Mechanical ventilation was used for patients associated with severe respiratory failure before surgery. All 110 cases were treated with antibiotics, and usage ceased for most of them when the acute inflammation was controlled.

\section{Comorbidity and morbidity}

Based on previous research, multiple rib fractures are easy to suffer from variety of complications. The major complications associated with thoracic trauma include hemothorax, pneumothorax, pulmonary contusion, pulmonary laceration, acute vascular injury, and abdominal solid-organ injury $(12,13)$. All such cases received prompt specialized treatment in our research.

\section{Treatment of the conservative group}

There were 51 patients who received conservative medical treatment, and the number of fractured ribs ranged from 2 to 12 . We use a pectoral girdle or external fixator for fixation of the fractured ribs. Additionally, we administered anti-inflammatories, maintained acid-base balance, and used aggressive pulmonary toilet, pain control and other treatments according to the different patient conditions. Closed thoracic drainage was treated for the patients associated hemothorax and/or pneumothorax. Mechanical ventilation was used for the patients with severe lung damage. Analgesic therapies were administered to patients without obvious analgesia contraindications. 

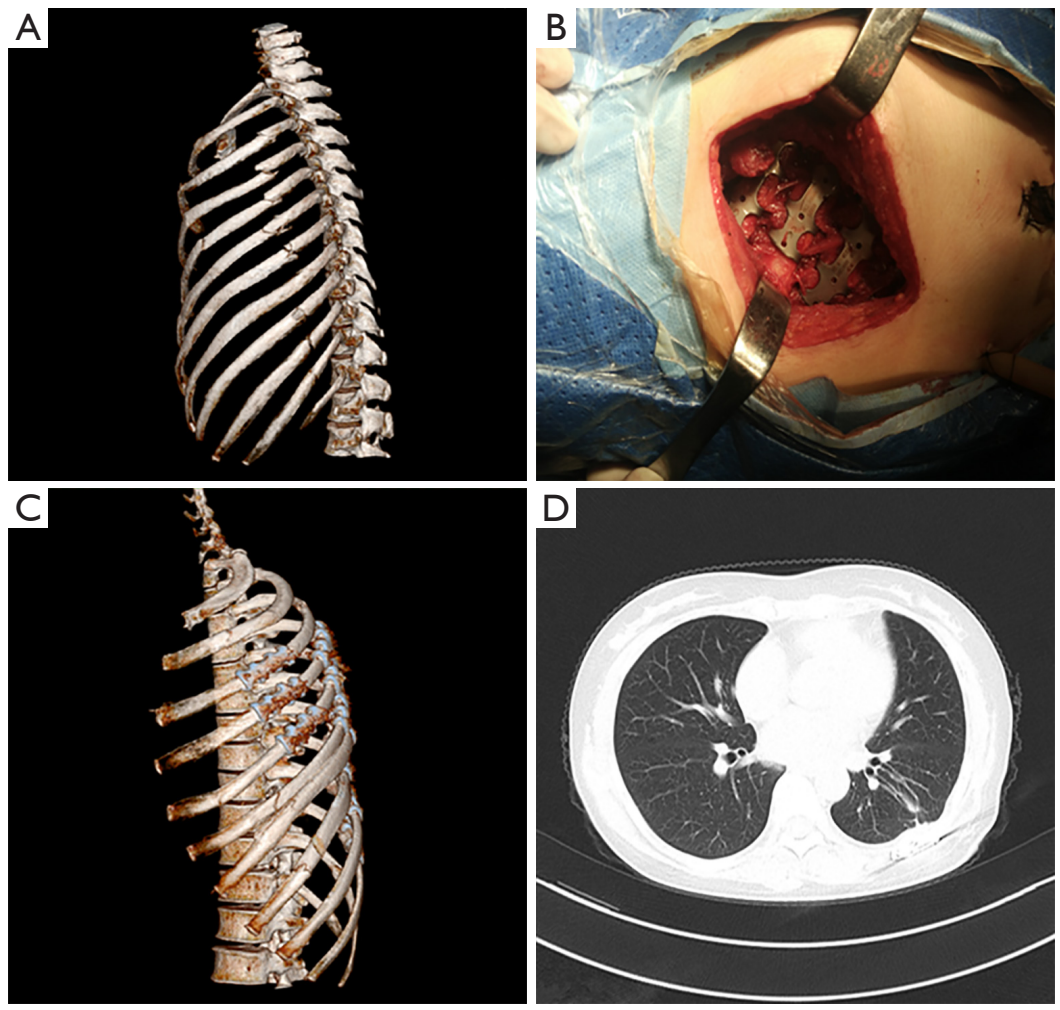

Figure 1 Clinical data of a typical case with multiple rib fractures. (A) Fractures after fixation; (B) the fractured ribs before surgery; (C) the fixed ribs after surgery; (D) CT scan of the patient $24 \mathrm{~h}$ after surgery. CT, computerized tomography.

\section{Surgical group}

In all 59 patients who underwent surgery, active bleeding and organ damage within the thorax were excluded first, and the hydrothorax was evacuated promptly. All the patients were treated using new pure titanium fixator plates (YLG02, WASTON, Changzhou, Jiangsu, China). The patients were placed in the lateral or horizontal position under general anesthesia. The selection of the appropriate surgical incision was based on the site and number of fractures. Generally, the length of the incision was between 5 and $15 \mathrm{~cm}$. After incision of the skin and subcutaneous tissue, blood clot around the fracture site was removed, the clavicular periosteum approximately $2 \mathrm{~cm}$ around fracture was lifted, and an anatomical reduction was performed. A suitable pure titanium fixator to place on the fracture was chosen, the rib concertrator fixation device was placed at the two sides of the fractured rib. Special pliers were used to further tighten the plate locking device (Figure 1). We did not attempt to fix all the fractured ribs, we fixed the ribs that supported the chest wall. For patients with severe associated complications, we chose to complete open-heart surgery first and then fix the fractured ribs. Analgesics were used to relieve the rib fracture pain.

\section{Statistical analysis}

Quantitative variables that were approximately normally distributed were compared using a two-sample $t$-test. Dichotomous data were compared using the Pearson $\chi^{2}$ or Fisher's exact test. All statistical analysis was performed using SPSS version 19.0. Continuous data are presented as the mean $\pm \mathrm{SD}$, and $\mathrm{P}<0.05$ was considered to indicate a significant difference.

\section{Results}

\section{The therapeutic results of the two groups}

A total of 110 patients with thoracic trauma were included in our study. According to the statistical analysis of the general characteristics, there was no significant difference in basic patients' information between the two groups (Table 1). Fifty-nine patients received surgical treatment, 
Table 1 The clinical characteristics of the patients

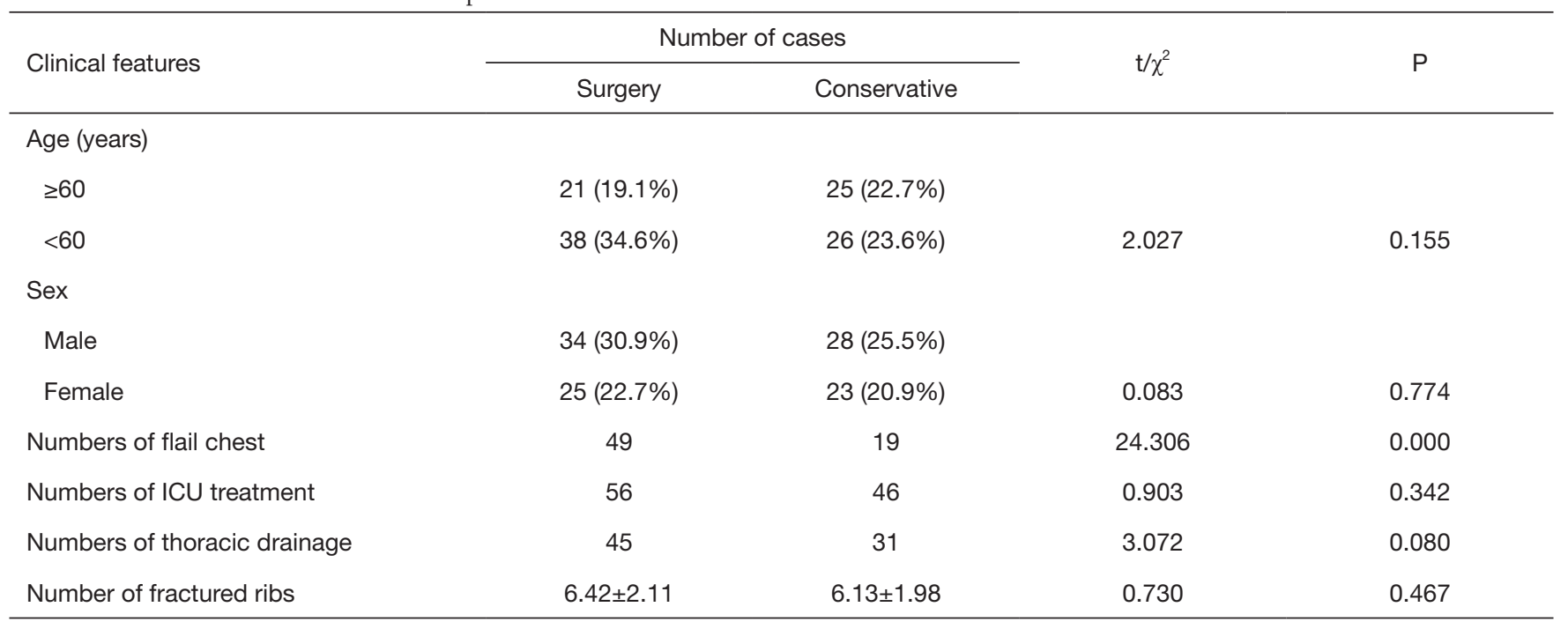

and 49 cases were diagnosed with flail chest in this group, we used new pure titanium plates to fix the fractured ribs, and the paradoxical respiratory movement was corrected. There were 3.7 plates (median, 5; range, 2-12) implanted per patient. The average duration of surgery was $85.2 \mathrm{~min}$, with a range from $42 \mathrm{~min}$ for the implantation of one plate to $150 \mathrm{~min}$ in a patient who received 12 plates, and Closed thoracic drainage was performed on the 49 patients with severe hemothorax and/or pneumothorax. After surgery, patients with flail chest had satisfactory correction, and the chest X-rays showed restored bilateral thoracic symmetry. We successfully followed up 52 cases 6 months after surgery, and all were assessed by chest X-ray or chest computed tomography (CT). We found that all the plates were firmly fixed without breakage or detachment. In 1 case, the plates were removed because of chronic pain 12 months after surgery. There were 51 patients in the conservative group, and 19 cases were diagnosed with flail chest, Mechanical ventilation was used for 10 patients with severe lung damage, and closed thoracic drainage was performed on the 34 patients with severe hemothorax and/ or pneumothorax. All the 51 patients recovered successfully and were discharged, including 1 who underwent clavicular fixation surgery.

\section{Clinical features of the two groups}

The statistical analysis examined hospital days, ICU stay days, indwelling thoracic drainage tube time, fracture displacement after admission, atelectasis after medical treatment, delayed hemopneumothorax, hospital costs, $\mathrm{PaO}_{2}$ of arterial blood and $\mathrm{PaCO}_{2}$ of arterial blood in the two groups of patients and compared the differences between the groups. We found that the patient arterial $\mathrm{PaO}_{2}$ and $\mathrm{PaCO}_{2}$ improved significantly after surgery and those patients in the surgery group required fewer ICU days and less thoracic drainage tube time. Fewer patients had associated delayed hemopneumothorax or atelectasis in the surgical treatment group, and the pain scores by VAS were lower in the surgical treatment group for the period following $24 \mathrm{~h}$ after treatment. However, the patients treated surgically had higher hospital costs than those treated conservatively. No significant differences regarding displacement occurred after treatment between the two groups (Tables 2,3, Figures 1,2).

\section{Mechanical ventilation for the conservative group patients}

There were 7 patients in the conservative group with associated severe pulmonary contusion, and mechanical ventilation was employed for these patients. We used intermittent positive pressure (IPPV) or continuous positive airway pressure (CPAP) ventilation mode to improve oxygenation. In addition, 2 patients who required long-term mechanical ventilation treatment underwent tracheotomy. Both were weaned successfully with obvious improvement of clinical symptoms and arterial $\mathrm{PaO}_{2}$ and slower respiratory rates. 
Table 2 Postoperative features

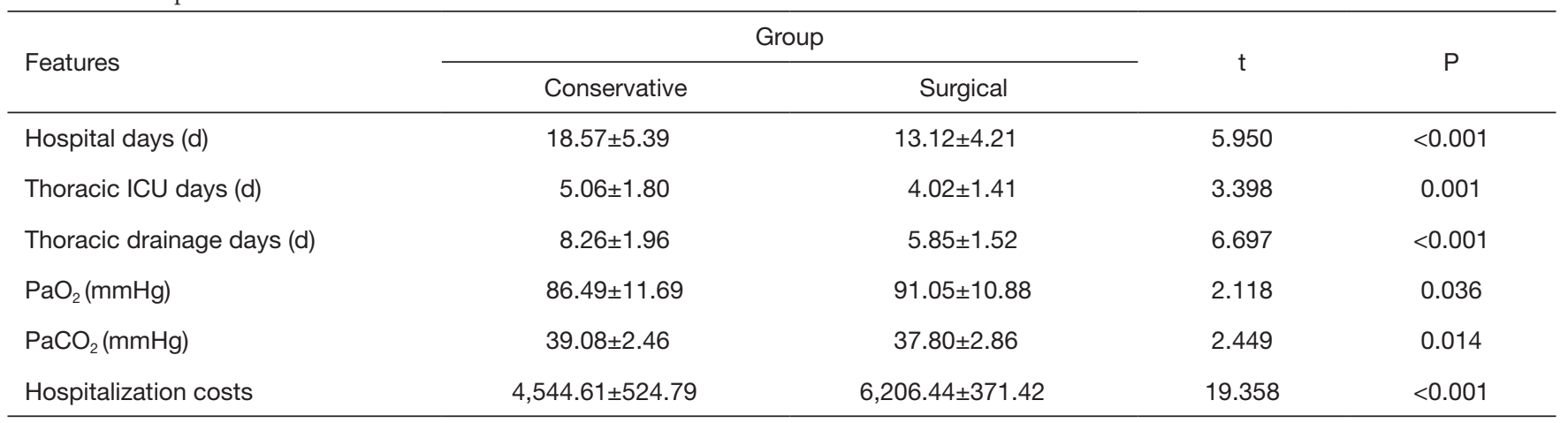

$\mathrm{ICU}$, intensive care unit; $\mathrm{PaO}_{2}$, partial pressure of arterial oxygen; $\mathrm{PaCO}_{2}$, partial pressure of carbon dioxide.

Table 3 Complications of two groups

\begin{tabular}{lccc}
\hline Groups & Displacement after treatment (cases) & Atelectasis after treatment (cases) & Delayed hemopneumothorax (cases) \\
\hline Surgical & 1 & 10 & 7 \\
Conservative & 2 & 17 & 16 \\
$\chi^{2}$ & 0.511 & 3.965 & 6.295 \\
$P$ & 0.475 & 0.046 & 0.012 \\
\hline
\end{tabular}

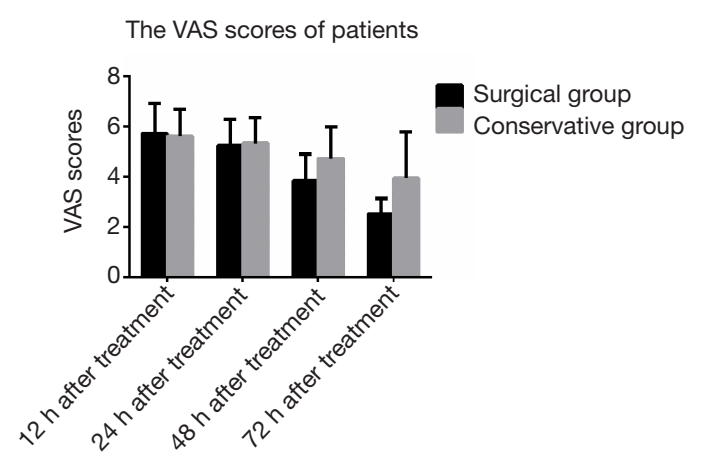

Figure 2 VAS scores of the patients in the two groups after treatment. VAS, visual analog scales.

\section{Discussion}

Multiple rib fractures can have a good prognosis if patients receive timely therapy. The selection of the therapy mode is an important factor regarding the therapeutic effects. This study assessed two different treatments for multiple rib fractures and compared the major features of the two groups. In our study, we found that the measures of $\mathrm{PaO}_{2}$ and $\mathrm{PaCO}_{2}$ improved significantly in the surgical group because of the difficulty of correcting thoracic deformities with conservative treatment and the decreased volume of the thoracic cavity (14). The complication rate was lower in the surgical group in our study; Swart's meta-analysis of rib fractures drew a similar conclusion (15). While there is also no lack of similar reports of different treatments for severe chest trauma, the specific VAS for pain were infrequently reported, whereas our study indicated that the patients in the surgery group had lower VAS scores and superior analgesia results. Additionally, although patients had higher hospitalization expenses in the surgical group in our study, the costs of therapy for long-term complications in the conservative group should also be considered.

Among the many traditional management methods for rib fractures, conservative treatment can avoid potential surgical complications such as foreign body reaction to the fixation material, injury to the intercostal neurovascular structures, and delayed healing of the surgical incision (16). However, nonoperative management may also lead to symptomatic malunion or nonunion. Compression dressings were always used for extensive floating of the chest wall, and they can influence the lung function of patients, particularly by restricting the normal lung ventilation function. In addition, the efficacy of fixation was not always satisfactory (17). The use of towel clips for traction of the ribs is another treatment for severe flail chest; however, 
obtaining a satisfactory curative effect is difficult. In recent years, growing evidence has suggested that surgery offered a reliable and effective therapy for severe rib fractures (18). Granetzny also reported that the operative group required significantly fewer mechanical ventilation, thoracic ICU and inpatient days than the conservative treatment group (19). For patients with associated severe soft issue contusions, repeated irrigation of the incision and standardized antisepsis can reduce the incidence of poor incisional healing effectively. Currently, with the development of minimally invasive medicine, video-assisted thoracoscopy has also been proven to be a safe and effective method for treating multiple rib fractures $(20,21)$. However, the outcomes of these treatments have not been described for specific injuries or regarding long-term quality of life.

Surgery is the predominant method of treatment for severe thoracic trauma (22). For patients with associated multiple fractures of multiple ribs, the fractured ribs should be fixed to improve the respiratory and circulatory function of the patients $(23,24)$. Several implants have been particularly designed for the fixation of fractured ribs. These implants include locking plates, splints (MatrixRIB, Synthes), U plates, claw-like plates, absorbable plates, Judet struts, mesh wrap, memory alloy embracing fixators and other plates $(22,25)$. Pure titanium plates have a competitive advantage in surgery for severe rib fractures; according to the bio-engineering research, pure titanium has good histocompatibility $(26,27)$. Moreover, these patients can undergo magnetic resonance (MR) scan or CT scan after surgery, with no need for plate removal for life. The (claw-like) pure titanium plates showed strong plasticity and do not require elevation of the rib periosteum. In addition, these plates have the advantage of simple manipulation, which can reduce the operative time and improve postoperative recovery. the pure titanium plate is lightweight, which can reduce the postoperative pain. But the all the plate has it's disadvantage currently used, the ideal material to perform osteosynthesis need good histocompatibility and strong enough for the first, then it should be easy to mold, because different people have different shape of ribs, for the last, it would be better if the material can absorb when the fracture ribs heal completely.

Traumatic asphyxia is a rare syndrome caused by blunt thoracoabdominal trauma, which clearly raises the intrathoracic pressure. It is often characterized by edema, subconjunctival hemorrhage and petechial hemorrhage of the face, neck and the upper parts of the thorax (28). Most patients will recover after conservative treatment. Kántor et al. reported a case with permanent visual loss (29). In our clinical experience, cases of traumatic asphyxia are often associated with several rib fractures. For these patients, we stabilized the respiratory and circulatory functions first. Closed thoracic drainage was promptly accomplished for the patients with pneumothorax, after which the fractured ribs were fixed by surgical or conservative treatment. For respiratory failure patients, the respiratory support mode of positive end-expiratory pressure (PEEP) is needed to open collapsed alveolar units. Nearly all the traumatic asphyxia patients had associated rib fracture and pulmonary contusion; thus, the therapy mode we tended to select for these patients was surgical treatment.

Pain management of multiple rib fractures is necessary throughout the full treatment course (30). Patients have difficulty sleeping and coughing up the secretions in the respiratory system because of pain, which can increase the incidence of pulmonary infection and prolong the hospital stay. The traditional treatment method for these symptoms is the short-term use of powerful pain relievers (31). In our study, we found that the early use of patient-controlled intravenous analgesia (PCIA) reduced the stress response and helped patients expectorate the secretions. The use of PCIA also minimizes the risk of pulmonary infection. For patients with serial rib fractures, fixation of the fractured ribs and correction of the paradoxical breathing can also alleviate pain. Compared with conservative treatment, surgical treatment of multiple rib fracture has the advantages of controlling chest wall floating and shorter disease duration, the latter resulting from the superior analgesic effect of PCIA in the surgical treatment group. Therefore, surgical treatment showed advantages over conservative treatment in pain control for multiple rib fractures.

The character and geometry of human ribs vary. A study showed that human ribs have a relatively thin (1-2 mm) cortex surrounding soft marrow (32) and that individual ribs do not have high stress tolerance. Surgical complications of rib fractures include superficial wound infections, pleural empyemas, plate loosening or wire migration, and fracture nonunion (33-35). From our research, surgical fixation can meaningfully improve the respiratory function of patients with severe rib fractures, and the paradoxical respiratory movement can be corrected. These results represent a unique single-center experience, and the research findings are likely limited due to the retrospective design of the study. Larger studies are required to further identify the benefits of rib fixation surgery. Large multi-center trials are 
needed to provide high evidence of better strategies for the treatment of severe thoracic trauma, which is also our next endeavor.

\section{Acknowledgements}

None.

\section{Footnote}

Conflicts of Interest: The authors have no conflicts of interest to declare.

Ethical Statement: This is a retrospective study, and the study was authorized by the hospital's ethics committee (2017L16). All patients were aware of pros and cons of the thoracic surgery; they gave a specific written informed consent.

\section{References}

1. Lema MK, Phillipo LC, Joseph BM, et al. Pattern and outcome of chest injuries at Bugando Medical Centre in Northwestern Tanzania. J Cardiothorac Surg 2011;6:7.

2. Holcomb JB, McMullin NR, Kozar RA, et al. Morbidity from rib fractures increases after age 45. J Am Coll Surg 2003;196:549-55.

3. Pettiford BL, Luketich JD, Landreneau RJ. The management of flail chest. Thorac Surg Clin 2007;17:25-33.

4. Kerr-Valentic MA, Arthur M, Mullins RJ, et al. Rib fracture pain and disability: Can we do better? J Trauma 2003;54:1058-63.

5. Doben AR, Eriksson EA, Denlinger CE, et al. Surgical rib fixation for flail chest deformity improves liberation from mechanical ventilation. J Crit Care 2014;29:139-43.

6. Cannon RM, Smith JW, Franklin GA, et al. Flail chest injury: are we making any progress? Am Surg 2012;78:398-402.

7. Nirula R, Mayberry JC. Rib Fracture Fixation: Controversies and Technical Challenges. Am Surg 2010;76:793-802.

8. Lafferty PM, Anavian J, Will RE, et al. Operative Treatment of Chest Wall Injuries: Indications, Technique, and Outcomes. J Bone Joint Surg Am 2011;93:97-110.

9. Muhm M, Härter J, Weiss C, et al. Severe trauma of the chest wall: surgical rib stabilization versus non-operative treatment. Eur J Trauma Emerg Surg 2013;39:257-65.

10. Witt CE, Bulger EM. Comprehensive approach to the management of the patient with multiple rib fractures: a review and introduction of a bundled rib fracture management protocol. Trauma Surg Acute Care Open 2017;2:e000064.

11. Chan EG, Stefancin E, Cunha JD. Rib Fixation Following Trauma: A Cardiothoracic Surgeon's Perspective. J Trauma Treat 2016;5:339.

12. Sirmali M, Turut H, Topcu S, et al. A comprehensive analysis of traumatic rib fractures: morbidity, mortality and management. Eur J Cardiothorac Surg 2003;24:133-8.

13. Chien CY, Chen YH, Han ST, et al. The number of displaced rib fractures is more predictive for complications in chest trauma patients. Scand J Trauma Resusc Emerg Med 2017;25:19.

14. Carver TW, Milia DJ, Somberg C, et al. Vital capacity helps predict pulmonary complications after rib fractures. J Trauma Acute Care Surg 2015;79:413-6.

15. Swart E, Laratta J, Slobogean G, et al. Operative Treatment of Rib Fractures in Flail Chest Injuries: A Meta-analysis and Cost-Effectiveness Analysis. J Orthop Trauma 2017;31:64-70.

16. Wu WM, Yang Y, Gao ZL, et al. Which is better to multiple rib fractures, surgical treatment or conservative treatment? Int J Clin Exp Med 2015;8:7930-6.

17. Majercik S, Wilson E, Gardner S, et al. In-hospital outcomes and costs of surgical stabilization versus nonoperative management of severe rib fractures. J Trauma Acute Care Surg 2015;79:533-8; discussion 538-9.

18. Marasco SF, Davies AR, Cooper J, et al. Prospective randomized controlled trial of operative rib fixation in traumatic flail chest. J Am Coll Surg 2013;216:924-32.

19. Granetzny A, Abd El-Aal M, Emam E, et al. Surgical versus conservative treatment of flail chest. Evaluation of the pulmonary status. Interact Cardiovasc Thorac Surg 2005;4:583-7.

20. Sing RF, Mostafa G, Matthews BD, et al. Thoracoscopic resection of painful multiple rib fractures: case report. J Trauma 2002;52:391-2.

21. Fraser SF, Tan C, Kuppusamy MK, et al. The role of a video-assisted thoracic approach for rib fixation. Eur J Trauma Emerg Surg 2017;43:185-90.

22. Richardson JD, Franklin GA, Heffley S, et al. Operative fixation of chest wall fractures: an underused procedure? Am Surg 2007;73:591-6.

23. Kasotakis G, Hasenboehler EA, Streib EW, et al. Operative fixation of rib fractures after blunt trauma: A practice management guideline from the Eastern Association for the Surgery of Trauma. J Trauma Acute 
Care Surg 2017;82:618-26.

24. Qiu M, Shi Z, Xiao J, et al. Potential Benefits of Rib Fracture Fixation in Patients with Flail Chest and Multiple Non-Flail Rib Fractures. Indian J Surg 2016;78:458-63.

25. Nirula R, Allen B, Layman R, et al. Rib fracture stabilization in patients sustaining blunt chest injury. Am Surg 2006;72:307-9.

26. Gulbahar G, Kaplan T, Gundogdu AG. et al. A Rare and Serious Syndrome That Requires Attention in Emergency Service: Traumatic Asphyxia. Case Rep Emerg Med 2015;2015:359814.

27. Berthet JP, Solovei L, Tiffet O, et al. Chest-wall reconstruction in case of infection of the operative site: is there any interest in titanium rib osteosynthesis? Eur J Cardiothorac Surg 2013;44:866-74.

28. Song J, Yan T, Wang T, et al. Internal fixation of claw-type rib bone plates on multiple fractured ribs. Int J Clin Exp Med 2017;10:6934-41.

29. Kántor T, Grigorescu B, Popescu G, et al. Traumatic

Cite this article as: Liu Y, Xu S, Yu Q, Tao Y, Peng L, Qi S, Han H, Chen M. Surgical versus conservative therapy for multiple rib fractures: a retrospective analysis. Ann Transl Med 2018;6(22):439. doi: 10.21037/atm.2018.10.12 asphyxia with permanent visual loss. Case report. Orv Hetil 2017;158:864-8.

30. Majercik S, Pieracci FM. Chest Wall Trauma. Thorac Surg Clin 2017;27:113-21.

31. Gordy S, Fabricant L, Ham B, et al. The contribution of rib fractures to chronic pain and disability. Am J Surg 2014;207:659-62.

32. Sales JR, Ellis TJ, Gillard J, et al. Biomechanical testing of a novel, minimally invasive rib fracture plating system. J Trauma 2008;64:1270-4.

33. Balci AE, Eren S, Cakir O, et al. Open fixation in flail chest: review of 64 patients. Asian Cardiovasc Thorac Ann 2004;12:11-5.

34. Mayberry JC, Kroeker AD, Ham LB, et al. Long-term morbidity, pain, and disability after repair of severe chest wall injuries. Am Surg 2009;75:389-94.

35. Nirula R, Diaz JJ Jr, Trunkey DD, et al. Rib Fracture Repair: Indications, Technical Issues, and Future Directions. World J Surg 2009;33:14-22. 